\title{
FIXED POINT THEOREMS FOR MAPPINGS SATISFYING INWARDNESS CONDITIONS
}

BY

\section{JAMES CARISTI}

ABSTRACT. Let $X$ be a normed linear space and let $K$ be a convex subset of $X$. The inward set, $I_{K}(x)$, of $x$ relative to $K$ is defined as follows: $I_{K}(x)=$ $\{x+c(u-x): c \geqslant 1, u \in K\}$. A mapping $T: K \rightarrow X$ is said to be inward if $T x \in$ $I_{K}(x)$ for each $x \in K$, and weakly inward if $T x$ belongs to the closure of $I_{K}(x)$ for each $x \in K$. In this paper a characterization of weakly inward mappings is given in terms of a condition arising in the study of ordinary differential equations. A general fixed point theorem is proved and applied to derive a generalization of the Contraction Mapping Principle in a complete metric space, and then applied together with the characterization of weakly inward mappings to obtain some fixed point theorems in Banach spaces.

0 . Introduction. Let $X$ be a topological vector space, $K \subset X$, and $T$ a mapping of $K$ into $X$. An inwardness condition on $T$ is one which asserts that, in some sense, $T$ maps points $x$ of $K$ "toward" $K$, or more precisely into the set generated by rays emanating from $x$ and passing through other points of $K$. Such conditions are always weaker than the assumption that $T$ map the boundary of $K$, $\partial K$, into $K$. They have been formulated in a variety of ways and imposed by several authors recently in connection with studies both in fixed point theory and in certain differential equations. Our purpose in this paper is to illustrate how different types of inwardness assumptions are related, and to prove several new fixed point theorems in which these concepts play a role.

Before stating precise definitions we give a brief review of some of the previous work in this area.

The study of inward mappings originated with the investigations of $\mathrm{B}$. Halpern in his 1965 doctoral thesis [7] where he obtained a generalization of the Schauder-Tychonov Theorem, a result he and Bergman further generalized in 1968 [9]. Since then many results have appeared in the literature concerning inward and weakly inward mappings in Halpern's sense, for both single and multivalued mappings (cf. [3] , [6] , [8], [9], [14], [16]-[19]).

Another type of inwardness assumption was used by $\mathrm{H}$. Brezis [1] in

Received by the editors May 1, 1974 and, in revised form, October 16, 1974.

AMS (MOS) subject classifications (1970). Primary 47H10; Secondary 54H25.

Key words and phrases. Fixed point theorems, contraction and nonexpansive mappings, inward and weakly inward conditions, complete metric space, Banach space. 
connection with his study of the differential equation

$$
x^{\prime}=f(x)
$$

where $x:[0, \infty) \rightarrow R^{n}$ and the mapping $f: K \subset R^{n} \rightarrow R^{n}$ is locally lipschitzian. Brezis proved that the condition

$$
\lim _{h \rightarrow 0^{+}} h^{-1} d(x+h f(x), K)=0 \quad \text { for all } x \in K
$$

(where $d(x, K)=\inf \{d(x, y): y \in K\}$ ) is equivalent to the flow invariance of $K$ in the sense that if $\varphi$ is a solution of $(0.1)$ and $\varphi\left(t_{0}\right) \in K$ for some $t_{0}$, then $\varphi(t) \in K$ for all $t \geqslant t_{0}$ in the interval of solution. Some of the work related to this result can be found in [5], [13], [15], [20]-[22]. We remark that a mapping satisfying $(0.2)$ with "lim" replaced by "lim inf" is called positively subtangential in [22].

Before turning to our principal fixed point theorem and its applications we give precise definitions of the inwardness assumptions used here and we show how our concepts are related to those of Brezis. Our first observation, in fact, shows that if $K$ is a convex subset of a normed linear space $X$, then $T: K \rightarrow X$ is weakly inward in Halpern's sense if and only if $f=I+T$ satisfies Brezis' condition (0.2). (Here $I$ denotes the identity mapping.) This connection yields as a consequence the fact that two of our principal fixed point results overlap known results of Martin [13] and Vidossich [21], a fact we comment upon in later remarks.

1. Characterization of weakly inward mappings. Let $X$ be a topological linear space and $K$ a subset of $X$. If $x \in K$ we define the inward set, $I_{K}(x)$, of $x$ with respect to $K$ as follows:

$$
I_{K}(x)=\{x+c(u-x): u \in K \text { and } c \geqslant 1\}
$$

We say that a mapping $T: K \rightarrow X$ is inward in case $T x \in I_{K}(x)$ for each $x \in K$. We say that $T$ is weakly inward in case $T x$ belongs to the closure of $I_{K}(x)$ for each $x \in K$.

Note that in the usual definition of $I_{K}(x)$ as in [9], the numbers $c$ were taken as simply nonnegative. However, in previous work $K$ has always been assumed to be convex, an assumption we will not always make, although (1.1) is equivalent to the usual definition of $I_{K}(x)$ in case $K$ is convex. We choose to require $c \geqslant 1$ for technical reasons which arise later. The following theorem is an essential tool in dealing with weakly inward mappings, and relates their study to the work of Brezis and others.

THEOREM 1.2. Let $K$ be a convex subset of a normed linear space $X$ and let $f: K \rightarrow X$. Then for each $x \in K$ 


$$
\lim _{h \rightarrow 0+} h^{-1} d(x-h f(x), K)=0
$$

if and only if $I-f$ is weakly inward.

Proof. Suppose (1.3) holds and let $x \in K$. Let $\epsilon>0$ be given. Then there is a number $h_{0}$ such that $0<h_{0}<1$ and $h_{0}^{-1} d\left(x-h_{0} f(x), K\right)<\epsilon / 2$. By definition of distance there exists $u \in K$ such that

$$
\left\|x-h_{0} f(x)-u\right\|<d\left(x-h_{0} f(x), K\right)+1 / 2 h_{0} \epsilon .
$$

Notice that $x+h_{0}^{-1}(u-x)$ belongs to $I_{K}(x)$. Since

$$
\begin{aligned}
\left\|\left[x+h_{0}^{-1}(u-x)\right]-[x-f(x)]\right\| & =h_{0}^{-1}\left\|u-\left(x-h_{0} f(x)\right)\right\| \\
& <h_{0}^{-1}\left[d\left(x-h_{0} f(x), K\right)+1 / 2 h_{0} \epsilon\right]<\epsilon,
\end{aligned}
$$

$(I-f)(x)$ is in the closure of $I_{K}(x)$ and hence $I-f$ is weakly inward.

Now suppose $I-f$ is weakly inward and let $x \in K$. Let $\epsilon>0$ be given.

Since $(I-f)(x)$ is in the closure of $I_{K}(x)$, choose a sequence $\left\{x_{n}\right\}$ in $I_{K}(x)$ such that $x_{n} \rightarrow(I-f)(x)$. Then there exists $N$ such that for all $n \geqslant N$,

Notice that

$$
\left\|x_{n}-(x-f(x))\right\|<\epsilon .
$$

$$
\begin{aligned}
& h^{-1} d(x-h f(x), K) \\
& \quad \leqslant h^{-1}\left\|[x-h f(x)]-\left[x+h\left(x_{N}-x\right)\right]\right\|+h^{-1} d\left(x+h\left(x_{N}-x\right), K\right) .
\end{aligned}
$$

Since $x_{N} \in I_{K}(x)$ and $K$ is convex, there exists $h_{0}>0$ such that $x+h_{0}\left(x_{N}-x\right)$ $\in K$. Thus if $0<h \leqslant h_{0}, h^{-1} d\left(x+h\left(x_{N}-x\right), K\right)=0$ and

$$
\begin{aligned}
h^{-1} d(x-h f(x), K) & \leqslant h^{-1}\left\|[x-h f(x)]-\left[x+h\left(x_{N}-x\right)\right]\right\| \\
& =\left\|x-f(x)-x_{N}\right\|<\epsilon,
\end{aligned}
$$

proving $\lim _{h \rightarrow 0+} h^{-1} d(x-h f(x), K)=0$. Q.E.D.

Corollary 1.4. If $f, K$, and $X$ are as in Theorem 1.2, then $f$ is weakly inward if and only if

$$
\lim _{h \rightarrow 0+} h^{-1} d((1-h) x+h f(x), K)=0 \quad \text { for all } x \in K .
$$

Observe that the convexity of $K$ was only used in the second half of Theorem 1.2, and in fact, without convexity the same proof can be used to obtain

Proposition 1.5. If $X$ is a normed linear space with $K \subset X$, and if $f$ : $K \rightarrow X$ has the property that $I-f$ is weakly inward, then

$$
\inf _{h>0}\left\{h^{-1} d(x-h f(x), K)\right\}=0 \quad \text { for all } x \in K .
$$

Proposition 1.6. If $X$ is a normed linear space with $K \subset X$, and if $f$ : $K \rightarrow X$ satisfies condition (1.3), then $I-f$ is weakly inward. 
Finally we remark that when $K$ is convex, condition $(0.2)$ is equivalent to the assumption that the mapping $I+f$ is weakly inward.

2. Fixed point theorems. Much of the recent work in fixed point theory has been directed toward determining when a mapping $T: K \rightarrow X$ for $K \subset X$ has a fixed point. It is not necessary always to assume that $T: K \rightarrow K$, but rather $T: \partial K \rightarrow K$ is often a sufficient assumption for existence of fixed points (e.g. [2]). A weaker assumption for convex $K$, the Leray-Schauder boundary condition, has been used by many authors in obtaining their results (e.g. [4], [12]). As previously mentioned, the Schauder-Tychonov Theorem is true for weakly inward mappings [9]. We are interested in generalizing two important theorems to weakly inward mappings: the Contraction Mapping Principle and Kirk's theorem for nonexpansive self-mappings of a bounded, closed, convex set with normal structure [11]. In the case of inward mappings, an extension of Kirk's theorem was obtained by S. Reich [17] by showing first [18] that an inward "condensing" mapping with bounded range defined on a closed, convex subset of a Banach space has a fixed point. His technique, however, apparently does not work for weakly inward mappings. We will prove first that a weakly inward contraction mapping always has a fixed point, and apply this to Reich's argument in [17] to reach our improvement of his extension of Kirk's theorem.

The important tool for all that follows is the next theorem, which will also give us a generalization of the Contraction Mapping Principle.

THEOREM 2.1. Let $(X, d)$ be a complete metric space, $K$ a closed subset of $X$. Suppose $f: K \rightarrow K$ is an arbitrary function and $T: K \rightarrow X$ is continuous. If there exists a real number $r<0$ such that

$$
d(f(x), T f(x)) \leqslant d(x, T x)+r d(x, f(x)) \text { for all } x \in K,
$$

then $f$ has a fixed point.

Proof. Let $\Gamma$ be the collection of ordinals strictly less than $\Omega$, the first uncountable ordinal. Let $\gamma_{0}$ be the first element of $\Gamma$. Let $x_{0} \in K$ and set $x_{\gamma_{0}}=x_{0}$. For fixed $\alpha \in \Gamma$ suppose that for all $\gamma \in \Gamma$ with $\gamma<\alpha$ we have defined $x_{\gamma} \in K$ in such a way that (i) if $\gamma=\gamma^{\prime}+1$ then $x_{\gamma}=f\left(x_{\gamma^{\prime}}\right)$ and (ii) if $\gamma_{n} \rightarrow \gamma$ then $x_{\gamma_{n}} \rightarrow x_{\gamma}$. To complete the induction we must define $x_{\alpha}$.

Suppose $\alpha=\alpha^{\prime}+1$. Since $\alpha$ cannot be the successor of anything but $\alpha^{\prime}$ we can define $x_{\alpha}=f\left(x_{\alpha^{\prime}}\right)$.

Suppose $\alpha_{n} \rightarrow \alpha$. To define $x_{\alpha}$ we must show that if $\beta_{n} \rightarrow \alpha$ then there is $x \in K$ such that $x_{\alpha_{n}} \rightarrow x$ and $x_{\beta_{n}} \rightarrow x$. By the well-ordering of $\Gamma$ we can define a sequence $\left\{\gamma_{n}\right\}$ consisting of all of the elements of $\left\{\alpha_{n}\right\} \cup\left\{\beta_{n}\right\}$ and which is nondecreasing.

Claim 1. If $\xi_{0}<\xi<\alpha$ then 


$$
d\left(x_{\xi}, T x_{\xi}\right) \leqslant d\left(x_{\xi_{0}}, T x_{\xi_{0}}\right)+r \sum_{\xi_{0} \leqslant \gamma<\xi} d\left(x_{\gamma}, x_{\gamma+1}\right) .
$$

The proof of Claim 1 is by transfinite induction on $\xi$. If $\xi=\xi_{0}+1$ we have

$$
d\left(x_{\xi}, T x_{\xi}\right)=d\left(f\left(x_{\xi_{0}}\right), T f\left(x_{\xi_{0}}\right)\right) \leqslant d\left(x_{\xi_{0}}, T x_{\xi_{0}}\right)+r d\left(x_{\xi_{0}}, x_{\xi}\right) .
$$

If $\xi=\eta+1$, and we know $\Sigma_{\xi_{0} \leqslant \gamma<\eta} d\left(x_{\gamma}, x_{\gamma+1}\right)<\infty$ and

$$
d\left(x_{\eta}, T x_{\eta}\right) \leqslant d\left(x_{\xi_{0}}, T x_{\xi_{0}}\right)+r \sum_{\xi_{0} \leqslant \gamma<\eta} d\left(x_{\gamma}, x_{\gamma+1}\right),
$$

then

$$
\begin{aligned}
d\left(x_{\xi}, T x_{\xi}\right) & =d\left(f\left(x_{\eta}\right), T f\left(x_{\eta}\right)\right) \leqslant d\left(x_{\eta}, T x_{\eta}\right)+r d\left(x_{\eta}, x_{\xi}\right) \\
& \leqslant\left[d\left(x_{\xi_{0}}, T x_{\xi_{0}}\right)+r \sum_{\xi_{0} \leqslant \gamma<\eta} d\left(x_{\gamma}, x_{\gamma+1}\right)\right]+r d\left(x_{\eta}, x_{\xi}\right) \\
& =d\left(x_{\xi_{0}}, T x_{\xi_{0}}\right)+r \sum_{\xi_{0} \leqslant \gamma<\xi} d\left(x_{\gamma}, x_{\gamma+1}\right) .
\end{aligned}
$$

Finally, if $\xi_{n} \rightarrow \xi$ and we know $\Sigma_{\xi_{0} \leqslant \gamma<\xi_{n}} d\left(x_{\gamma}, x_{\gamma+1}\right)<\infty$, and

$$
d\left(x_{\xi_{n}}, T x_{\xi_{n}}\right) \leqslant d\left(x_{\xi_{0}}, T x_{\xi_{0}}\right)+r \sum_{\xi_{0} \leqslant \gamma<\xi_{n}} d\left(x_{\gamma}, x_{\gamma+1}\right)
$$

for all $n$, then let $s_{n}=\Sigma_{\xi_{0} \leqslant \gamma<\xi_{n}} d\left(x_{\gamma}, x_{\gamma+1}\right)$ and $s=\Sigma_{\xi_{0} \leqslant \gamma<\xi} d\left(x_{\gamma}, x_{\gamma+1}\right)$. Since $0 \leqslant d\left(x_{\xi_{0}}, T x_{\xi_{0}}\right)+r s_{n}$, we see that $\left\{s_{n}\right\}$ is bounded. Clearly $\left\{s_{n}\right\}$ is nondecreasing and $s_{n} \leqslant s$ for each $n$. Hence $\lim _{n \rightarrow \infty} s_{n} \leqslant s$. If $\left\{\delta_{i}\right\}$ is an enumeration of $\left\{\gamma: \xi_{0} \leqslant \gamma<\xi\right\}$, then $s=\lim _{n \rightarrow \infty} \sum_{i=1}^{n} d\left(x_{\delta_{i}}, x_{\delta_{i}+1}\right)$. For each $n$ choose $m$ so large that $\xi_{m} \geqslant \max \left\{\delta_{1}, \ldots, \delta_{n}\right\}$. Thus $s_{m} \geqslant \sum_{i=1}^{n} d\left(x_{\delta_{i}}, x_{\delta_{i}+1}\right)$ and hence $s \leqslant \sup \left\{s_{m}\right\}=\lim _{m \rightarrow \infty} s_{m} \leqslant s$. Therefore, $s$ is finite and $s_{n} \rightarrow s$ as $n \rightarrow$ $\infty$. By the continuity of $T$ we obtain

$$
d\left(x_{\xi}, T x_{\xi}\right) \leqslant d\left(x_{\xi_{0}}, T x_{\xi_{0}}\right)+r \sum_{\xi_{0} \leqslant \gamma<\xi} d\left(x_{\gamma}, x_{\gamma+1}\right),
$$

proving the claim.

Claim 2. If $\xi_{n}<\alpha$ for $n=0,1, \ldots$ and $\left\{\xi_{n}\right\}$ is nondecreasing, and if we define

then $\Sigma \epsilon_{n}<\infty$.

$$
\epsilon_{n}=d\left(x_{\xi_{n-1}}, T x_{\xi_{n-1}}\right)-d\left(x_{\xi_{n}}, T x_{\xi_{n}}\right)
$$

For the proof of this claim observe that for each $n$, Claim 1 gives

$$
\epsilon_{n} \geqslant d\left(x_{\xi_{n-1}}, T x_{\xi_{n-1}}\right)-\left[d\left(x_{\xi_{n-1}}, T x_{\xi_{n-1}}\right)+r \sum_{\xi_{n-1}<\gamma<\xi_{n}} d\left(x_{\gamma}, x_{\gamma+1}\right)\right] \geqslant 0 \text {. }
$$

Hence $\left\{d\left(x_{\xi_{n}}, T x_{\xi_{n}}\right)\right\}$ is a nonincreasing sequence. Since $\Sigma_{1}^{n} \epsilon_{i}=d\left(x_{\xi_{0}}, T x_{\xi_{0}}\right)-$ $d\left(x_{\xi_{n}}, T x_{\xi_{n}}\right)$, we get

$$
\sum_{1}^{\infty} \epsilon_{i}=d\left(x_{\xi_{0}}, T x_{\xi_{0}}\right)-\lim _{n \rightarrow \infty} d\left(x_{\xi_{n}}, T x_{\xi_{n}}\right)
$$

which proves the claim. 
The following claim involves the sequence $\left\{\gamma_{n}\right\}$ defined earlier.

Claim 3. $d\left(x_{\gamma_{n}}, x_{\gamma_{n+1}}\right) \leqslant \Sigma_{\gamma_{n} \leqslant \gamma<\gamma_{n+1}} d\left(x_{\gamma}, x_{\gamma+1}\right)$.

We proceed by transfinite induction on $\gamma_{n+1}$. If $\gamma_{n+1}=\gamma_{n}+1$, then $d\left(x_{\gamma_{n}}, x_{\gamma_{n+1}}\right)=d\left(x_{\gamma_{n}}, x_{\gamma_{n}+1}\right)$. If $\gamma_{n+1}=\eta+1$ and

$$
d\left(x_{\gamma_{n}}, x_{\eta}\right) \leqslant \sum_{\gamma_{n} \leqslant \gamma<\eta} d\left(x_{\gamma}, x_{\gamma+1}\right)
$$

then

$$
\begin{aligned}
d\left(x_{\gamma_{n}}, x_{\gamma_{n+1}}\right) & \leqslant d\left(x_{\gamma_{n}}, x_{\eta}\right)+d\left(x_{\eta}, x_{\gamma_{n+1}}\right) \\
& \leqslant \sum_{\gamma_{n} \leqslant \gamma<\eta} d\left(x_{\gamma}, x_{\gamma+1}\right)+d\left(x_{\eta}, x_{\gamma_{n+1}}\right) \\
& =\sum_{\gamma_{n}<\gamma<\gamma_{n+1}} d\left(x_{\gamma}, x_{\gamma+1}\right) .
\end{aligned}
$$

Finally, suppose $\xi_{i} \rightarrow \gamma_{n+1}$ as $i \rightarrow \infty$, and that for each $i$,

$$
d\left(x_{\gamma_{n}}, x_{\xi_{i}}\right) \leqslant \sum_{\gamma_{n} \leqslant \gamma<\xi_{i}} d\left(x_{\gamma}, x_{\gamma+1}\right) \text {. }
$$

Let $\epsilon>0$. Then there exists a number $N$ such that for all $i \geqslant N, d\left(x_{\xi_{1}}, x_{\gamma_{n+1}}\right)$ $<\epsilon$. Hence

$$
\begin{aligned}
d\left(x_{\gamma_{n}}, x_{\gamma_{n+1}}\right) & \leqslant d\left(x_{\gamma_{n}}, x_{\xi_{N}}\right)+d\left(x_{\xi_{N}}, x_{\gamma_{n+1}}\right) \\
& \leqslant \sum_{\gamma_{n}<\gamma<\xi_{N}} d\left(x_{\gamma}, x_{\gamma+1}\right)+\epsilon \\
& \leqslant \sum_{\gamma_{n}<\gamma<\gamma_{n+1}} d\left(x_{\gamma}, x_{\gamma+1}\right)+\epsilon,
\end{aligned}
$$

which completes the proof of Claim 3.

Now by Claims 3 and 1 ,

$$
d\left(x_{\gamma_{n}}, x_{\gamma_{n+1}}\right) \leqslant \sum_{\gamma_{n} \leqslant \gamma<\gamma_{n+1}} d\left(x_{\gamma}, x_{\gamma+1}\right) \leqslant-r^{-1} \epsilon_{n},
$$

where $\epsilon_{n}=d\left(x_{\gamma_{n}}, T x_{\gamma_{n}}\right)-d\left(x_{\gamma_{n+1}}, T x_{\gamma_{n+1}}\right)$.

By Claim 2, $\left\{x_{\gamma_{n}}\right\}$ is a Cauchy sequence, so there exists $x \in K$ such that $x_{\gamma_{n}} \rightarrow x$; hence $x_{\alpha_{n}} \rightarrow x$ and $x_{\beta_{n}} \rightarrow x$. Therefore by transfinite induction we have defined a subset $K_{1}$ of $K$ as $K_{1}=\left\{x_{\gamma}: \gamma \in \Gamma\right\}$. Let $m=\inf \{d(x, T x)$ : $x \in K_{1}$ \}.

Choose a sequence $\left\{x_{\gamma_{i}}\right\}$ in $K_{1}$ such that $\left\{\gamma_{i}\right\}$ is increasing and $d\left(x_{\gamma_{i}}, T x_{\gamma_{i}}\right)$ $\rightarrow m$. Since $\left\{\gamma_{i}\right\}$ is countable, it cannot converge to $\Omega$, or else $\Omega$ would be a countable ordinal. Since $\left\{\gamma_{i}\right\}$ is increasing, it must converge to its least upper bound, say $\gamma<\Omega$. Hence $d\left(x_{\gamma}, T x_{\gamma}\right)=m$. But $\gamma+1 \in \Gamma$ and

$$
d\left(x_{\gamma+1}, T x_{\gamma+1}\right)=d\left(f\left(x_{\gamma}\right), T f\left(x_{\gamma}\right)\right) \leqslant d\left(x_{\gamma}, T x_{\gamma}\right)+r d\left(x_{\gamma}, f\left(x_{\gamma}\right)\right),
$$


implying that $d\left(x_{\gamma}, f\left(x_{\gamma}\right)\right)=0$ by the minimality of $m$. Q.E.D.

Let $X$ be a complete metric space and $K \subset X$. We say that a mapping $T$ : $K \rightarrow X$ is metrically inward if for each $x \in K$ there exists an element $u$ of $K$ such that

$$
d(x, u)+d(u, T x)=d(x, T x)
$$

where $u=x$ if and only if $x=T x$. For $X$ a normed linear space this assumption is weaker than the definition of inward given in $\S 1$.

THEOREM 2.2. Suppose $(X, d)$ is a complete metric space, $K$ a closed subset of $X$, and $T: K \rightarrow X$ a metrically inward contraction mapping with Lipschitz constant $k<1$. Then $T$ has a fixed point.

Proof. Suppose $T$ has no fixed point. Then it is possible to define $f$ : $K \rightarrow K$ as follows: $f(x)=u$ where $u \neq x$ is any element of $K$ such that $d(x, u)+$ $d(u, T x)=d(x, T x)$. For $x \in K$ then

$$
\begin{aligned}
d(f(x), T f(x)) & \leqslant d(f(x), T x)+d(T x, T f(x)) \\
& =d(x, T x)-d(x, f(x))+d(T x, T f(x)) \\
& \leqslant d(x, T x)+(k-1) d(x, f(x)) .
\end{aligned}
$$

Since $k-1<0$ we can apply Theorem 2.1 to obtain a fixed point for $f$, thus contradicting the definition of $f$. Q.E.D.

In a normed linear space, if a mapping is inward, then it is metrically inward. Thus we observe that if $X$ is a Banach space, $K$ a closed subset of $X$, and $T$ : $K \rightarrow X$ an inward contraction mapping, then $T$ has a fixed point.

There is a rather natural way of defining weakly inward mappings in a metric sense. It is still unknown whether Theorem 2.2 remains true under such a weaker assumption. As another application of Theorem 2.1, however, we will prove that Theorem 2.2 is true for a weakly inward contraction mapping defined on a closed, convex subset of a Banach space. In view of Theorem 1.2 this result is actually included in an observation by Martin [13, Proposition 3]. However, Martin derives his result from the theory of ordinary differential equations, a technique totally unlike our approach.

THEOREM 2.3. Let $X$ be a Banach space and $K$ a closed, convex subset of $X$. Let $T: K \rightarrow X$ be a weakly inward contraction mapping with Lipschitz constant $k<1$. Then $T$ has a fixed point.

Proof. Suppose $T$ has no fixed point. Choose $\epsilon>0$ so small that $k<$ $(1-\epsilon) /(1+\epsilon)$. We will define a function $f: K \rightarrow K$ such that for every $x \in K$ and for fixed $r<0$

$$
\|f(x)-T f(x)\|<\|x-T x\|+r\|x-f(x)\| .
$$


By Theorem 2.1 such a function $f$ has a fixed point, and the resulting contradiction completes the proof. In order to define $f$ it suffices to show that given $x \in K$ there exists $y \in K$ such that

$$
\|y-T y\|<\|x-T x\|+\left(k-\frac{1-\epsilon}{1+\epsilon}\right)\|x-y\| .
$$

Given $x \in K$, use Corollary 1.4 to obtain $h \in(0,1)$ such that

$$
h^{-1} d((1-h) x+h T x, K)<\epsilon\|x-T x\| .
$$

By the definition of distance, there exists an element $y$ of $K$ such that

$$
\|\bar{x}-y\|<h \epsilon\|x-T x\|, \quad \text { where } \bar{x}=(1-h) x+h T x .
$$

Observe also that $\|\bar{x}-x\|=h\|x-T x\|$, and (2.4) yields

$$
\frac{\|x-y\|}{\|\bar{x}-x\|} \leqslant \frac{\|x-\bar{x}\|+\|\bar{x}-y\|}{\|\bar{x}-x\|}=1+\frac{\|\bar{x}-y\|}{\|\bar{x}-x\|}<1+\epsilon .
$$

Thus

$$
\|\bar{x}-x\|>(1+\epsilon)^{-1}\|x-y\| .
$$

Using (2.4), (2.5), and the fact that $\epsilon-1<0$,

$$
\begin{aligned}
\|y-T y\| & \leqslant\|y-\bar{x}\|+\|\bar{x}-T x\|+\|T x-T y\| \\
& \leqslant\|y-\bar{x}\|+\|\bar{x}-T x\|+k\|x-y\| \\
& =\|y-\bar{x}\|+\|x-T x\|-\|x-\bar{x}\|+k\|x-y\| \\
& <\epsilon\|\bar{x}-x\|+\|x-T x\|-\|x-\bar{x}\|+k\|x-y\| \\
& =\|\bar{x}-x\|(\epsilon-1)+\|x-T x\|+k\|x-y\| \\
& <\frac{\|x-y\|}{1+\epsilon}(\epsilon-1)+\|x-T x\|+k\|x-y\| \\
& =\|x-T x\|+\left(k-\frac{1-\epsilon}{1+\epsilon}\right)\|x-y\| . \text { Q.E.D. }
\end{aligned}
$$

A mapping $T: K \rightarrow X$ is said to be pseudo-contractive if for all $x, y \in K$ and all $r>0$,

$$
\|x-y\| \leqslant\|(1+r)(x-y)-r(T x-T y)\| .
$$

$T$ is said to be nonexpansive if for all $x, y \in K$,

$$
\|T x-T y\| \leqslant\|x-y\| \text {. }
$$

The class of pseudo-contractive mappings is easily seen to include the nonexpansive mappings. Interest in the study of pseudo-contractive mappings stems from the fact that the mapping $T$ is pseudo-contractive if and only if $I-T$ is accretive [10].

The next theorem extends Kirk's theorem [11] to weakly inward mappings. 
In this theorem we assume that $K$ has the fixed point property for nonexpansive self-maps, i.e., that every nonexpansive mapping $T: K \rightarrow K$ has a fixed point. This is the case, for instance, if $K$ is weakly compact and has normal structure [11].

We should also remark that $G$. Vidossich has claimed a theorem [21, Theorem 5 (ii)] which, again in view of Theorem 1.2, is equivalent to Theorem 2.6 below. He uses differential equations techniques also.

THEOREM 2.6. Let $X$ be a Banach space, $K$ a closed, convex subset of $X$ which possesses the fixed point property for nonexpansive self-maps, and suppose $T: K \rightarrow X$ is a lipschitzian, pseudo-contractive mapping which is weakly inward. Then $T$ has a fixed point in $K$.

Proof. Choose $r>0$ so small that $k T$ is a contraction mapping where $k=r /(r+1)$. Define $B=[I+r(I-T)]^{-1} . B$ is single-valued and nonexpansive, for

$\|[I+r(I-T)] u-[I+r(I-T)] v\|=\|(1+r)(u-v)-r(T u-T v)\| \geqslant\|u-v\|$.

The range of $B$ must be contained in $K$. We will show that the domain of $B$ contains $K$. Let $z \in K$ be fixed and define $\widetilde{T}: K \rightarrow X$ by $\widetilde{T} x=k T x+(1-k) z$. $\widetilde{T}$ is easily seen to be a contraction mapping. Notice further that for each $x \in K$, $I_{K}(x)$ is a convex set containing $K$, hence so is the closure of $I_{K}(x)$. Therefore $\widetilde{T} x$ is a convex combination of elements of the closure of $I_{K}(x)$ and thus $\widetilde{T}$ is weakly inward. By Theorem 2.3 there exists $x^{*} \in K$ such that $\widetilde{T} x^{*}=x^{*}$. Hence

$$
x^{*}=k T x^{*}+(1-k) z=r(r+1)^{-1} T x^{*}+(r+1)^{-1} z .
$$

This implies $z=(r+1) x^{*}-r T x^{*}=x^{*}+r(I-T) x^{*}$. Hence $z$ is in the range of $I+r(I-T)$, so the domain of $B$ contains $K$. Therefore $B$ restricted to $K$ is a nonexpansive self-mapping of $K$, which must have a fixed point, say $x$. Then $x+r(x-T x)=x$ and thus $x=T x$. Q.E.D.

3. Remarks. After this paper was written the following more general formulation of Theorem 2.1 was suggested by Felix Browder (see James Caristi and W. A. Kirk, Geometric fixed point theory and inwardness conditions (to appear in Proc. Conference on the Geometry of Metric and Linear Spaces, Mich. State Univ., 1974)).

THEOREM (2.1)'. Let $(M, d)$ be a complete metric space and $f: M \rightarrow M$. If there exists a lower semicontinuous function $\varphi$ mapping $M$ into the nonnegative real numbers such that

$$
d(x, f(x)) \leqslant \varphi(x)-\varphi(f(x)), \quad x \in M,
$$

then $f$ has a fixed point.

The proof given for Theorem 2.1 establishes the above version upon replac- 
ing $d(x, T x)$ with $-\varphi(x) / r$ and making obvious modifications.

We very recently discovered that a theorem announced by I. Ekeland [C. R. Acad. Sci. Paris Sér. A-B 275 (1972), 1057-1059 (Théorème 1)] is equivalent to Theorem (2.1)'. Ekeland's result (which is not formulated as a fixed point theorem) is an abstraction of a lemma due to Bishop and Phelps. Earlier Felix Browder had suggested a possible connection between Theorem (2.1)' and the BishopPhelps approach and devised a simpler proof. Subsequently Chi Song Wong simplified our transfinite induction approach and W. A. Kirk observed that a proof of Theorem (2.1)' is implicit in a recent paper of A. Brфnsted [Pacific J. Math. 55 (1974), 335-341]. Finally we remark that further applications of Theorem (2.1)' have been given by Kirk and Caristi [Bull. Acad. Polon. Sci. 32 (1975)] and by Kirk [Proc. Seminar on Fixed Point Theory and its Applications, Dalhousie University, 1975 (to appear)].

The author is greatly indebted to Professor W. A. Kirk for his valuable assistance. The results of this paper comprise a portion of the author's University of Iowa doctoral dissertation.

\section{REFERENCES}

1. H. Brezis, On a characterization of flow-invariant sets, Comm. Pure Appl. Math. 23 (1970), 261-263. MR 41 \#2161.

2. F. E. Browder, Nonlinear mappings of nonexpansive and accretive type in Banach spaces, Bull. Amer. Math. Soc. 73 (1967), 875-882. MR 38 \#581.

3. - The fixed point theory of multi-valued mappings in topological vector spaces, Math. Ann. 177(1968), 283-301. MR 37 \#4679.

4. Semicontractive and semiaccretive nonlinear mappings in Banach spaces, Bull. Amer. Math. Soc. 74 (1968), 660-665. MR 37 \#5742.

5. M. G. Crandall, A generalization of Peano's existence theorem and flow invariance, Proc. Amer. Math. Soc. 36 (1972), 151-155. MR 46 \#5708.

6. Ky Fan, Extensions of two fixed point theorems of F. E. Browder, Math. Z. 112 (1969), 234-240. MR 40 \#4830.

7. B. R. Halpern, Fixed point theorems for outward maps, Doctoral Thesis, Univ. of California, Los Angeles, Calif., 1965.

8. - Fixed-point theorems for set-valued maps in infinite dimensional spaces, Math. Ann. 189 (1970), 87-98. MR 42 \#8357.

9. B. R. Halpern and G. M. Bergman, A fixed-point theorem for inward and outward maps, Trans. Amer. Math. Soc. 130 (1968), 353-358. MR 36 \#4397.

10. T. Kato, Nonlinear semigroups and evolution equations, J. Math. Soc. Japan 19 (1967), 508-520. MR 37 \#1820.

11. W. A. Kirk, A fixed point theorem for mappings which do not increase distances, Amer. Math. Monthly 72 (1965), 1004-1006. MR 32 \#6436.

12. - Fixed point theorems for nonexpansive mappings satisfying certain boundary conditions, Proc. Amer. Math. Soc. 50 (1975), 143-149.

13. R. H. Martin, Jr., Differential equations on closed subsets of a Banach space, Trans. Amer. Math. Soc. 179 (1973), 399-414. MR 47 \#7537.

14. W. V. Petryshyn and P. M. Fitzpatrick, Fixed point theorems for multivalued noncompact inward maps (to appear).

15. R. M. Redheffer, The theorems of Bony and Brezis on flow-invariant sets, Amer. Math. Monthly 79 (1972), 740-747. MR 46 \#2166. 
16. S. Reich, Fixed points in locally convex spaces, Math. Z. 125 (1972), 17-31. MR 46 \#6110.

17. - Remarks on fixed points, Atti Accad. Naz. Lincei Rend. Cl. Sci. Fis. Mat. Natur. (8) 52 (1972), 689-697. MR 48 \#9473.

18. - Fixed points of condensing functions, J. Math. Anal. Appl. 41 (1973), 460-467. MR 48 \#971.

19. - Fixed points of non-expansive functions, J. London Math. Soc. (2) 7 (1973), 5-10. MR 48 \#4855.

20. G. Vidossich, Existence comparison and asymptotic behavior of solutions of or dinary differential equations in finite and infinite dimensional Banach spaces (to appear).

21. Nonexistence of periodic solutions of differential equations and applications to zeros of nonlinear operators (to appear).

22. J. A. Yorke, Differential inequalities and non-Lipschitz scalar functions, Math. Systems Theory 4 (1970), 140-153. MR 42 \#3373.

DEPARTMENT OF MATHEMATICS, UNIVERSITY OF IOWA, IOWA CITY, IOWA 52242

Current address: Department of Mathematics, Texas Lutheran College, Seguin, Texas 78155 